\title{
COLLAGEN CHITOSAN SCAFFOLDS ON INDUCED SKIN DEFECT IN A RAT MODEL (AN EXPERIMENTAL STUDY)
}

\section{Salma A. Aboulgheit ${ }^{1}$ MSc., Sally M. Abdelkader² Ph.D., Moustafa N. Aboushelib ${ }^{2}$ Ph.D., Radwa A. Mehanna ${ }^{3}$ Ph.D., Enas M. Omar ${ }^{4}$ Ph.D., Yomna M. Ibrahim ${ }^{5}$ BDS.}

\section{ABSTRACT}

BACKGROUND: Over the past ten years, an alternative for maxillofacial reconstruction was offered through regenerative medicine. It provided a new scope for improving the reconstruction of the oral and maxillofacial structures whether they are hard tissues which include the teeth and bone or the soft tissues like the oral mucosa, skin, nerves and blood vessels.

OBJECTIVES: The aim of this study is to evaluate the effect of the collagen chitosan scaffolds on the duration and quality of skin surface healing.

MATERIALS AND METHODS: An experimental in vivo comparative study was conducted, on twelve adult male Sprague-Dawley rats, where an induced skin wound was made on the backs of rats and then classified into two groups; 1-control group and 2-scaffold group. The effect of the collagen-chitosan scaffolds on the duration and quality of skin surface healing were assessed using Masson's trichrome staining for collagen fibers and the imageJ software to calculate \% area after wound healing also the number of CD 68 macrophages was counted using histological sections then the two groups were compared using student t-test $(\mathrm{p}<0.05)$.

RESULTS: The scaffold group showed accelerated wound healing in comparison to the control group and with a significant result. Quality of healed skin was significantly better in the scaffold treated group, which is attributed to the better collagen alignment and deposition in the stained histological sections indicated by the \% area after wound healing. The number of CD 68 macrophages was higher in the scaffold group compared to the control group.

CONCLUSION: The collagen chitosan scaffolds can be considered as a treatment regimen for treating skin wounds.

KEYWORDS: maxillofacial reconstruction, collagen chitosan scaffold, skin surface healing, wound/healing.

${ }^{1}$ Assistant Lecturer of Dental Biomaterials, Faculty of Dentistry, Alexandria University, Egypt.

2 Professor of Dental Biomaterials, Faculty of Dentistry, Alexandria University, Egypt.

${ }^{3}$ Assistant Professor of Physiology, Faculty of Medicine, Alexandria University, Egypt.

${ }^{4}$ Lecturer of Oral Pathology Department, Faculty of Dentistry, Alexandria University, Egypt.

${ }^{5}$ Demonstrator of Dental Biomaterials, Faculty of Dentistry, Alexandria University, Egypt.

*Corresponding author

salmaabolgheit@yahoo.com

\section{INTRODUCTION}

Maxillofacial reconstruction has always relied on reshaping of the lost tissues due to accidents, traumas or tumors using tissue grafts from the same patient giving the tissues a close to natural appearance and some regaining of function. However, due to the increased esthetical demands of patients and the need to decrease the need of tissue grafts due to donor side morbidities, alternatives to the standard regimen of craniofacial reconstruction needed to be found (1).

Within the past ten years, a new alternative for the standard treatment of tissue regeneration of maxillofacial tissues has evolved. It offers a supplementary methodology for the reconstruction of the maxillofacial and oral tissues whether they are hard tissues which include the teeth and bone or the soft tissues like the oral mucosa, skin, nerves and blood vessels (2).
The skin is the largest organ of the body, which is directly faced by the surrounding environment. Thus, the skin is liable to various damages $(3,4)$. Acute or chronic wounds are considered to be a major clinical obstacle. Nowadays, the standard treatment methodology for full-thickness skin wounds is through transplantation or cell therapy (5). However, with the dermis either damaged or non-existent, the wound will not be epithelialized, and complete healing will not be achieved (6). The new tissue engineering methodology could open a new scope for the regeneration and repair of the skin. Until now, many products that were synthesized by tissue engineering are applied to treat skin defects. A perfect substitute of the dermis should form a barrier against microbes, keep the wound area moist, be porous to allow gas exchange and remove exudates (7). The substitute should also be biocompatible and non-adherent in 
order to be easily removed without damaging the newly formed tissues. It should also be made from a readily available biomaterial that is easy to process and possesses antibacterial properties to enhance wound healing (8).

A vast array of biomaterials has been used to treat skin defects including collagen, alginate, hyaluronic acid, chitosan, and fibrin glue. Collagen is the major protein in the extra cellular matrix (ECM) with a unique molecular structure that contributes to the extracellular scaffolding. Therefore, collagen is considered a crucial protein in supporting the tissues and maintaining the homeostasis of the ECM (9).

Additionally, collagen does not stimulate immunogenicity, it is biocompatible, biodegradable, possesses a porous structure and has the ability to maintain cellular structure and functions $(10,11)$. Owing to these characteristic capabilities, collagen shows promising results when used as a scaffold in tissue engineering. However, collagen scaffolds are not mechanically strong, and they are also unstable when hydrated which makes them contraindicated as scaffolds for certain tissues. In order to improve the mechanical properties, the collagen can be crosslinked with other natural or synthetic polymers through physical or chemical methods to create hybrid scaffolds that acquire the advantages of its forming compounds and eliminating their drawbacks when used separately (12).

One of the very promising candidates to be blended with collagen is chitosan. Chitosan is a natural biopolymer containing an amino group (-NH2) with an adjacent hydroxyl group. $(-\mathrm{OH})$. It also forms part of the hyaluronic acid as it possesses acetylglucosamine and glucosamine units. Chitosan is highly biocompatible and can be totally degraded enzymatically into a saccharide known as chitin. It has various applications in drug delivery and as a wound dressing material since its structure and function resemble those of the skin mucopolysaccharides $(13,14)$. Chitosan is considered as a promotor of wound healing and reepithelization of the tissues, an adhesive material for cellular attachment and proliferation and a non-immunogenic compound which will not be rejected by the natural tissues $(15,16)$.

This study aims to test the effect of applying collagen chitosan scaffolds to induced skin defects in a rat model and evaluate the wound healing in a rat model between a scaffold treated group and a control group. The null hypothesis states that there is no difference in wound healing time and skin integrity between the two groups.

\section{MATERIALS AND METHODS}

This study was conducted after receiving the approval of the ethics committee of the Faculty of Dentistry - Alexandria University.

\section{Grouping}

Twelve adult 8 weeks old male Sprague Dawley rats were divided into two groups. The two groups are:

1. Control group (1)
Six rats with twelve induced skin defects left to heal alone.

Subgroup 1A: three rats were sacrificed after forty-eight hours for immunohistochemical analysis.

Subgroup 1B: the remaining three rats were sacrificed after two weeks for functional assessment.

\section{Scaffold group (2)}

Six rats with twelve induced skin defects left to heal with scaffold in place.

Subgroup 2A: three rats were sacrificed after forty-eight hours for immunohistochemical analysis.

Subgroup 2B: remaining three rats were sacrificed after two weeks for functional assessment.

\section{I- Scaffold Fabrication}

\section{1- Preparation and verification of Collagen}

The procedure was done in the CERRMA lab, Alexandria Faculty of Medicine. Skin of newborn lambs was removed, rinsed in distilled water, and stored at $4^{\circ} \mathrm{C}$ until purification. The skin specimens were washed with ice-cold distilled water and any hair or wool was removed with a hair removal cream. After that the skin samples have been scraped clean of the fat and the connective tissue. Then the skin samples were washed thoroughly in ice-cold distilled water and sliced into $10 \times 10 \mathrm{~mm}$ pieces with a blade (17).

\section{Removal of non-collagenous solubilized material}

At $4^{\circ} \mathrm{C}$, the whole collagen type I isolation was conducted, and all the reagents were adjusted to this temperature before their use. $1.5 \mathrm{~mL}$ of ice-cold $0.5 \mathrm{M}$ sodium acetate together with the lamb skin samples $(0.5 \mathrm{~g}$ each) were placed into Eppendorfs $(1.5 \mathrm{ml})$ to eliminate the non-collagenous soluble proteins and polysaccharides. The Eppendorfs were triturated for 1 minute at $6 \mathrm{~m} / \mathrm{s}$ using an appropriate amalgamator (Softly 8, de Götzen, Italy). Supernatants were discarded and replenished with fresh $0.5 \mathrm{M}$ sodium acetate. This procedure was repeated for a total of seven cycles. Remaining sodium acetate was eliminated by washing the skin samples with ice-cold distilled water. Afterwards, the skin samples were then compressed to eliminate any extra liquid and placed into fresh Eppendorfs for further processing (17).

\section{COL1 extraction}

The skin samples were rinsed with $0.075 \mathrm{M}$ sodium citrate buffer for 1 minute at $6 \mathrm{~m} / \mathrm{s}$. Supernatants were removed then fresh buffer was added. Without removing the sodium citrate buffer, the skin samples were then subjected to six sequential 1-minute cycles $(6 \mathrm{~m} / \mathrm{s})$ of agitation. A clear and thick supernatant which represents COL1 was observed and collected. A final agitation cycle was done with a fresh sodium citrate buffer added to each dermal skin sample. All extracted supernatants of the COL1 were collected then centrifuged at $3200 \mathrm{~g}$ at $4^{\circ} \mathrm{C}$ for 10 minutes to remove any residues, and the clarified samples were transferred to 100,000 MWCO Amicon Ultra-15 centrifugal filter devices (Millipore). The centrifugation was continued at the same weight and temperature for 30 minutes for COL1 purification. The resultant collagen in the top compartments was placed in Eppendorfs and stored at $4^{\circ} \mathrm{C}$ (17). 
Verification was done using the UV spectrophotometer (DS11 FX, DeNovix, USA) and Transmission Electron microscope (TEM) (JEOL-100CX, Japan) to view the collagen type 1 fibrils.

\section{2-Chitosan production}

The procedure was done in the dental biomaterials department, Alexandria Faculty of Dentistry. Impurities were removed from marine shrimp shells (purchased from an Alexandrian fish market, Smouha, Alexandria, Egypt) using boiling water then the shells were placed in a hot-air oven (Hobersal, Spain) at $90 \circ \mathrm{C}$ for 6 hours to dry. In order to produce chitin and chitosan, a blender was used to homogenize the shells into small portions less than 15 pieces per mesh. The homogenized shells were kept frozen until used (18).

\section{Demineralization}

$1 \mathrm{~L}$ of $1 \mathrm{M} \mathrm{HCl}$ were added to 100 grams of shrimp shells in a beaker which was stirred at room temperature at $250 \mathrm{rpm}$ for 1 hour using a magnetic stirrer (F91T, Falc, Italy). To achieve a neutral $\mathrm{PH}$, the demineralized shells filtrated then washed with distilled water. Afterwards, the demineralized shells were bleached by adding ethanol for ten minutes and were dried in an oven at $70^{\circ} \mathrm{C}(18)$.

\section{Deproteinization}

The dried demineralized shells were added to $1 \mathrm{M} \mathrm{NaOH}$ at a solid/liquid ratio of $1: 10(\mathrm{~g} / \mathrm{mL})$. The procedure was performed under stirring for three hours at $80 \circ \mathrm{C}$. To obtain a neutral $\mathrm{PH}$, the solid was then filtered and washed with distilled water. For more bleaching, the dried demineralized shells were placed in ethanol for ten minutes, then the resulting chitin was dried in an oven at $70^{\circ} \mathrm{C}(18)$.

\section{Chitosan Production}

The resultant chitin underwent deacetylation by mixing it with $12.5 \mathrm{M} \mathrm{NaOH}$ at a solid/liquid ratio of $1: 15(\mathrm{~g} / \mathrm{mL})$. The resultant mixture was kept in an ultra-freezer at $-83 \circ \mathrm{C}$ overnight to cool down. Then, raising of the temperature of the mixture was done by raising the temperature to $115{ }^{\circ} \mathrm{C}$, and the reaction was further completed with stirring at 250 rpm for six hours. To reach a neutral PH the formed chitosan was rinsed, filtered with distilled water. The chitosan was then dried in an oven at $70 \circ \mathrm{C}(18)$. Verification of chitosan was done using Fourier-Transform Infrared Spectroscopy (FTIR) (Alpha II, Bruker, Germany).

\section{3-Collagen-chitosan scaffold fabrication}

Solutions of both collagen and chitosan were dissolved separately in $0.5 \mathrm{~mol} / \mathrm{L}$ acetic acid solution to prepare a $0.5 \%$ solution of each component. A mixture was made of the collagen and chitosan solutions with the ratio of 9:1 respectively. The collagen-chitosan mixture with the specified ratio was injected into a Teflon mold and 0.5\% glutaraldehyde was added to the mixture to act as a cross linker. The formed mixture was placed in a refrigerator for two hours at $-20^{\circ} \mathrm{C}$ and placed in a lyophilizer for 24 hours to be processed into a collagen-chitosan porous scaffold (19).
The scaffold was characterized using Scanning Electron microscope (SEM) (JEOL, JSM-5, Japan) and FTIR. Confocal microscopy (Zeiss LSM 510) was used for imaging of the scaffolds by inducing autofluorescence with a 488-nm argon laser and a long-pass 505nm filter was used to collect signals.

\section{II- Surgical Procedure}

Rats were purchased and housed in the Center of Excellence for Research in Regenerative Medicine and Applications (CERRMA). Rats were first anaesthetized using IM injections with a combination of $30 \mathrm{mg} / \mathrm{kg}$ body weight of ketamine and $5 \mathrm{mg} / \mathrm{kg}$ body weight of xylazine $\mathrm{HCl}$. The back skin of the rat was shaved then $10 \%$ povidone-iodine was used to disinfect the skin before a square-shaped excisional full-thickness skin wound $(1 \mathrm{~cm} \times 1 \mathrm{~cm})$ was conducted using a sterile scalpel. Group I which is the Control group (CG) was a negative control where skin injuries were left to heal on their own without intervention. Group 2 which is the collagen chitosan (CC) treated group, wounds were to be treated with collagen chitosan scaffold. To avoid further injuries or wound disturbance, each rat was housed in a separate cage. After surgery, IM antibiotics (Cefotax $100 \mathrm{mg} / \mathrm{mL}$ ) were given to all rats.

To visualize and follow up the wound healing process, digital images were captured, and wound area measurements were recorded. The wound healing process has been estimated based on the remaining wound area.

\section{Evaluation of the wound healing process}

Photographing of the skin wounds was done using a digital camera. Every three days the surface area of the wound was recorded for all the rats of both groups using a digital caliper. Photographing of the skin wounds started from the day of wound induction (day 0) till the wounds completely healed. Wound closure time was recorded for statistical comparison and the data was represented as mean and standard deviation.

Staining of the collagen fibers with Masson's Trichrome Stain

The histological sections were first deparaffinized, rehydrated then stained with Masson's trichrome stain to demonstrate the collagen fibers. A micrograph of each sample was captured at 200x magnification and the collagen fibers were stained with blue color using methyl blue dye. According to Pereira et al (20), the ratio of the collagen in the dermis, which is stained in green or blue, could be calculated compared to the total area of the slide using the ImageJ software (NIH, USA). The ratio of the blue pixels exceeding the threshold compared to the total pixels in the micrograph was used to calculate the percentage of collagen (\% area after wound healing) in each sample in order to compare between the groups.

\section{Histological and immunohistochemical study}

A sharp scalpel was used to obtain wedge shaped biopsies which include the central granulation tissue and the peripheral normal tissue. The wedge-shaped biopsies were collected from each group and per group. All the biopsies 
were kept in $10 \%$ buffered formalin, paraffin embedded, then sliced into $5 \mu \mathrm{m}$ thick sections.

In order to detect the CD68+ macrophages, the biopsy sections were deparaffinized and then rehydrated with descending grades of alcohol. A solution of 3\% hydrogen peroxide was used to inhibit the endogenous activity of peroxidase. After antigen retrieval, anti-CD68 primary antibody (mouse monoclonal Ab, clone KiM6, 1:100 dilution) and horseradish peroxide-conjugated secondary antibody (rabbit IgG, 1: 100 dilution) were used. DAB (3,3'-diaminobenzidine) (Lab Vision Corporation, Neo Markers, Fremont, USA) was used to detect the bound antibodies. In all runs, positive and negative controls were included. Finally, slides were counterstained with hematoxylin, mounted on a slide, and cover slipped. The macrophage infiltration was evaluated semi-quantitatively using a bright-field light microscope coupled with a digital camera. The number of infiltrated macrophages was counted by examining ten high power random microscopic fields in each specimen and the numbers were represented as mean and standard deviation.

Statistical Analysis

Statistical Analysis:

After collecting the data, it was tabulated and presented by using range (minimum and maximum), mean, standard deviation and median. Afterwards, a comparison between the two study groups was done using the student ttest to detect significant differences in the wound healing time between the two groups, the \% area after wound healing and the number of macrophages found in the injury site of both. The data was processed using SPSS 22 software (SPSS Inc., Chicago, IL, USA) with a significance level of $5 \%$ $(\mathrm{P}<0.05)$.

\section{RESULTS}

\section{Collagen verification}

The morphology of the prepared collagen was viewed using TEM (x1200) showing the spindle-like collagen type I fibrils homogenously distributed without agglomeration. The average length of the fibrils was $5.5 \mu \mathrm{m}$ and the thickness 39 $\mathrm{nm}$ (Figure 1A). A drop of the collagen solution was scanned by UV spectrophotometer in the range of $140-400 \mathrm{~nm}$ and the maximum absorption peak was found to be at the $230 \mathrm{~nm}$ wavelength, which is characteristic for $\mathrm{C}=\mathrm{O}, \mathrm{COOH}$, CONH2 bonds found in the collagen chains (Figure $1 \mathrm{~B}$ ).

\section{Verification of chitosan}

The structure of chitosan was verified using FTIR to identify characteristic functional groups in the range of $(4000 \mathrm{~cm}-1$ to $450 \mathrm{~cm}-1)$.

The stretching vibrations of $-\mathrm{OH}$ bond were found at $3429.57 \mathrm{~cm}-1$ and of $\mathrm{C}-\mathrm{H}$ were found at $2922.53 \mathrm{~cm}-1$. The absorption peaks at $1646.75 \mathrm{~cm}-1,1567.90 \mathrm{~cm}-1$, $1418.87 \mathrm{~cm}-1,1328.69 \mathrm{~cm}-1$ were associated with the presence of the $\mathrm{C}=\mathrm{O}$ stretching of the amide I band, bending vibrations of the $\mathrm{N}-\mathrm{H}$ (N-acetylated residues, amide II band), $\mathrm{C}-\mathrm{H}$ bending and $\mathrm{OH}$ bending respectively. The peak at $1080.83 \mathrm{~cm}^{-1}$ was attributed to the $\mathrm{C}-\mathrm{O}$ stretching (Figure 2).

\section{Verification of Collagen-Chitosan Scaffold}

The SEM micrograph shows the interwoven porous structure of the collagen-chitosan scaffold with mean pore diameter $90 \mu \mathrm{m}$ of and mean porosity of $66.4 \%$ (Figure 3A).

The FTIR spectrum of the scaffold shows the bonds and peaks found in the originally forming molecules without additional bands. These bonds originated from the interaction of the collagen and chitosan through hydrogen bonds without any alterations in the structure and properties of the original molecules. As chitosan is added to the collagen, the bands representing amide I (1658 cm-1) and amide III $(1243 \mathrm{~cm}-1)$ show a decrease in intensity contrary to the bands of amide II $(1563 \mathrm{~cm}-1)$ which an increased intensity compared to the chitosan spectrum (Figure 3B).

The confocal microscope coupled with the auto fluorescence technique confirmed the presence of the collagen fibers (red), the chitosan (green) and the merging of both collagen and chitosan in the scaffold (Figure 3C-E).

Wound healing in days

Table (1) shows a comparison between the two studied groups according to wound healing in days. The control group showed a mean wound healing in about 30 days compared with the scaffold treated group which healed in almost 12 days. These results show a significant difference between the two groups $(p<0.001)$ as the scaffold treated group needed less than half time required for the control group to heal. The wound induction and size for both control and study groups at days 7 and 12 as well as the complete healing is shown in figure 4 A-F.

Trichrome staining of the two groups after complete healing

Table (2) shows a comparison between the two studied groups according to \% area (after complete healing). The numbers indicate the amount of collagen deposition in the wound area which was higher in the test group (31.64) compared to the control group (19.43) showing a significant increase in the scaffold treated group. A histological confirmation of the collagen deposition is shown in Figure 5 after staining of the sections with Masson's trichrome stain. Wedge shaped biopsies were taken including the normal skin with the healed skin as indicated by the triangle in figure 5A. Figure 5B shows a low magnification image of the border between the normal tissue and healed skin. The sections show the arrangement of the collagen fibers at the beginning of the test for normal skin (Figure 5C) and after complete healing (Figure 5D) showing a regaining of skin integrity and normal structure.

Macrophage cell count for the two groups after $\mathbf{4 8}$ hours Table (3) shows a comparison between the two studied groups according to macrophage cell count (after 48 hours). A higher macrophage cell count was found in the scaffold treated group (40.0) in contrast to the control group (24.7). These results also comply with the immunohistochemical 
images (Figure 6) showing the presence of the CD 68 macrophages and their abundance in the test group as an indication of immunoreactivity after 48 hours.

Table (1): Comparison between the two studied groups according to wound healing in days

\begin{tabular}{|c|c|c|c|c||}
\hline $\begin{array}{c}\text { Wound } \\
\text { healing }\end{array}$ & $\begin{array}{c}\text { Control } \\
(\mathbf{n}=6)\end{array}$ & $\begin{array}{c}\text { Group 2 } \\
(\mathbf{n}=6)\end{array}$ & $\mathbf{t}$ & $\mathbf{p}$ \\
\hline Min. - Max. & $29.0-31.0$ & $12.0-14.0$ & & \\
Mean \pm SD. & $30.0 \pm 0.94$ & $12.60 \pm 0.84$ & $43.500^{*}$ & $<0.001$ \\
Median (IQR) & $30.0(29.0-$ & $\begin{array}{c}12.0(12.0- \\
13.0)\end{array}$ & & \\
\hline
\end{tabular}

t: Student t-test

$\mathrm{p}$ : $\mathrm{p}$ value for comparing between the studied groups

*: Statistically significant at $\mathrm{p} \leq 0.05$

Table (2): Comparison between the two studied groups according to \% Area (after complete healing)

\begin{tabular}{|c|c|c|c|}
\hline $\begin{array}{c}\text { \% Area } \\
\text { (complete } \\
\text { healing) }\end{array}$ & $\begin{array}{c}\text { Control } \\
(n=6)\end{array}$ & $\begin{array}{c}\text { Group } 2 \\
(n=6)\end{array}$ & \\
\hline Min. - Max. & $\begin{array}{c}17.22- \\
22.29\end{array}$ & $\begin{array}{l}29.40- \\
33.79\end{array}$ & \\
\hline Mean \pm SD. & $19.43 \pm 1.86$ & $31.64 \pm 1.62$ & $34^{*}<0.001^{*}$ \\
\hline Median (IQR) & $\begin{array}{c}19.30(17.9- \\
20.6)\end{array}$ & $\begin{array}{c}31.69(30.4- \\
32.9)\end{array}$ & \\
\hline
\end{tabular}

t: Student t-test

$\mathrm{p}$ : $\mathrm{p}$ value for comparing between the studied groups

*: Statistically significant at $\mathrm{p} \leq 0.05$

Table (3): Comparison between the two studied groups according to macrophages (after 48 hours)

\begin{tabular}{|c|c|c|c|c||}
\hline $\begin{array}{c}\text { Macrophages } \\
\text { (48 hours) }\end{array}$ & $\begin{array}{c}\text { Control } \\
(\mathbf{n}=\mathbf{6})\end{array}$ & $\begin{array}{c}\text { Group 2 } \\
(\mathbf{n}=\mathbf{6})\end{array}$ & $\mathbf{t}$ & $\mathbf{p}$ \\
\hline Min. - Max. & $19.0-30.0$ & $35.0-42.0$ & & \\
Mean \pm SD. & $24.67 \pm 4.32$ & $40.0 \pm 2.61$ & $7.443^{*}$ & $<0.001^{*}$ \\
Median (IQR) & $\begin{array}{c}24.5(21.0- \\
29.0)\end{array}$ & $\begin{array}{c}40.5(40.0- \\
42.0)\end{array}$ & & \\
\hline
\end{tabular}

t: Student t-test

$\mathrm{p}$ : $\mathrm{p}$ value for comparing between the studied groups

*: Statistically significant at $\mathrm{p} \leq 0.05$

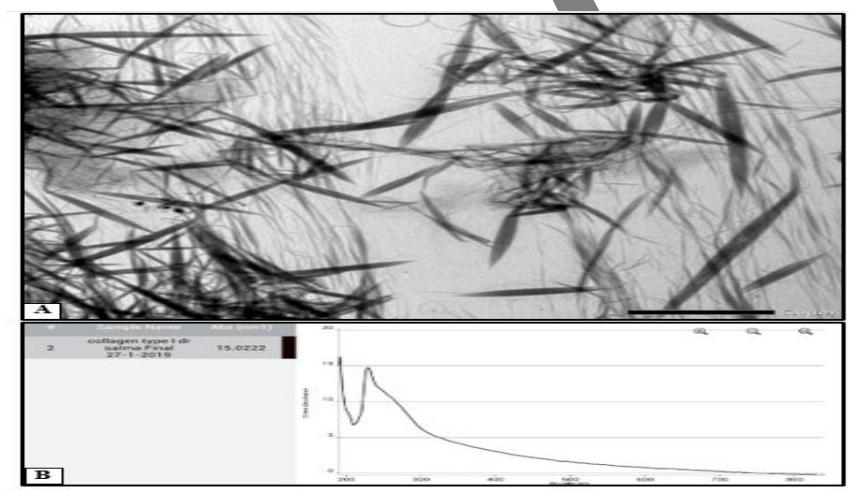

Figure (1): a) TEM of collagen fibers, b) UV verification of collagen showing the peak at 230 .

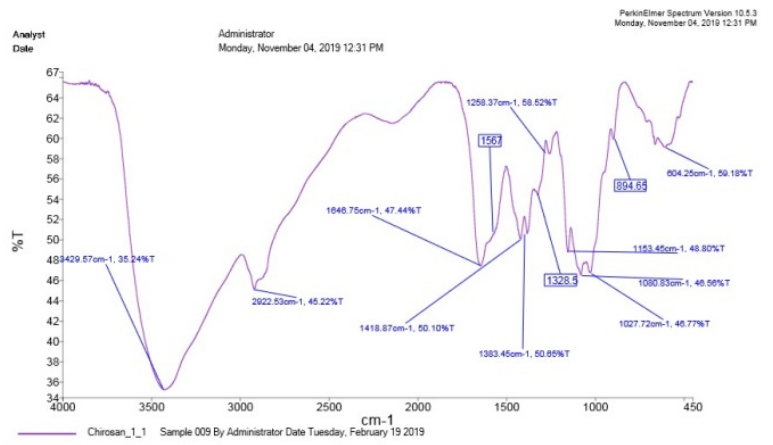

Figure (2): FTIR of chitosan,

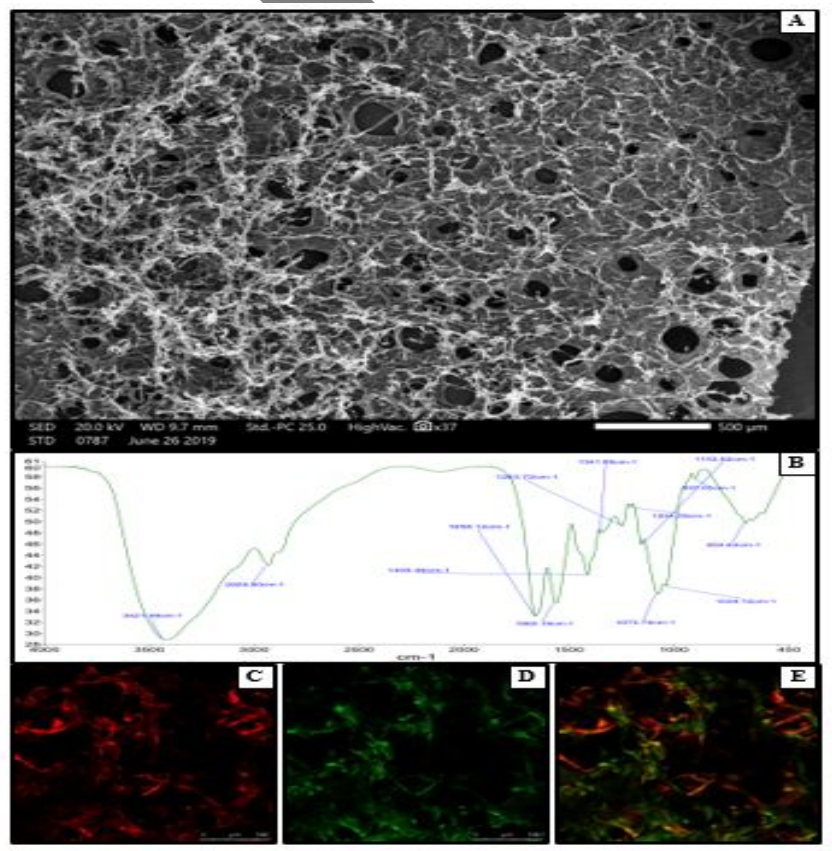

Figure (3): a) SEM of collagen chitosan scaffold, b) FTIR of collagen chitosan scaffold, c) Auto-fluorescence showing collagen, d) Auto-fluorescence showing chitosan, e) Autofluorescence showing merged collagen chitosan.

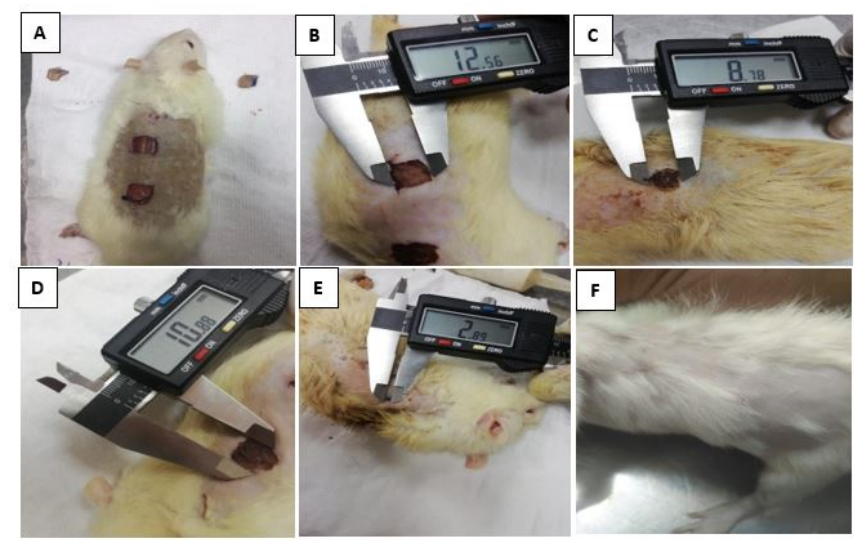


Figure (4): a) Day 0 for both groups, b) Day 7 control group, c) Day 7 group 2, d) Day 12 control group, e) Day 12 group 2, f) Complete healing for both groups.

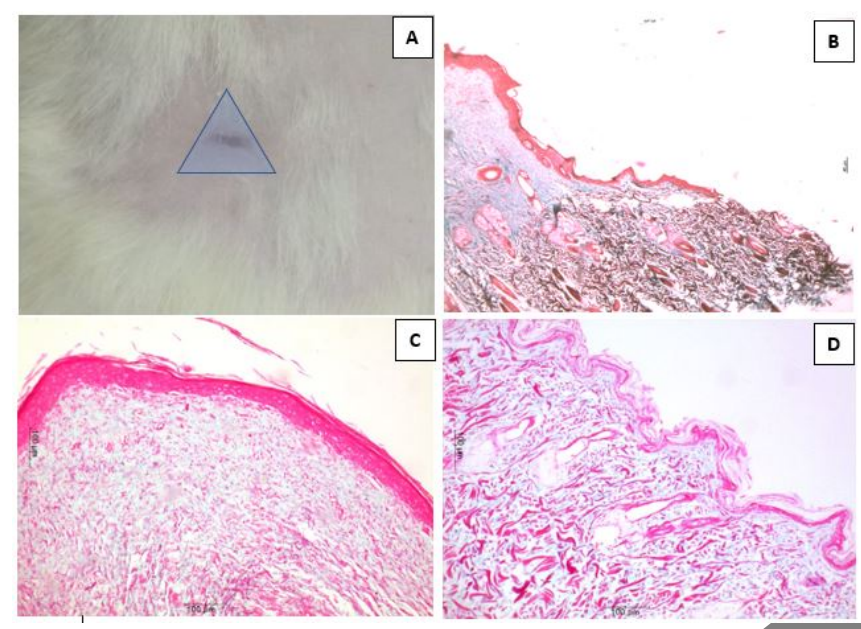

Figure (5): a) Diagram showing position of obtaining the biopsy b) Photomicrograph showing the interface between the normal tissue and the wound healing border (magnification $\mathrm{x} 100$ ) c, d) Photomicrographs of skin biopsies after injury and left for complete healing showing variable amounts and arrangement of collagen fibers in the dermis (magnification x200). Figures b, c and d are Masson's trichrome-stained representative sections.

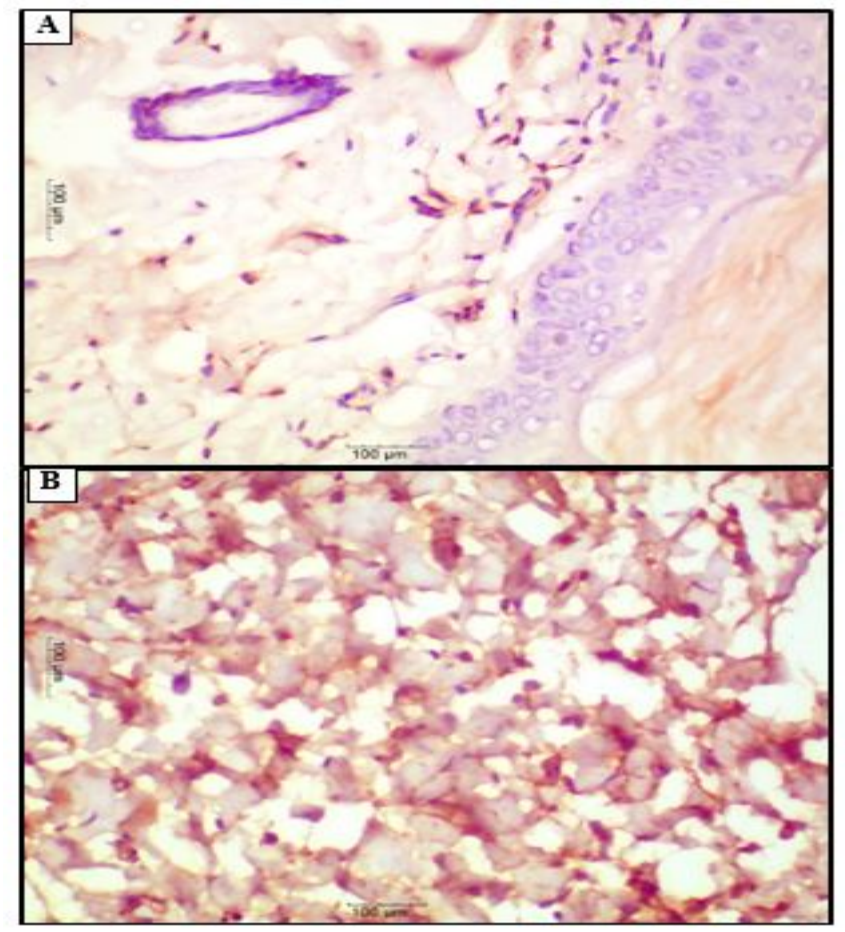

Figure (6): Immunohistochemical photomicrograph showing variable degrees of immunoreactivity of CD 68 macrophages after 48 hours. (Brown color). Each figure is hematoxylin-counterstained. (X400).

\section{DISCUSSION}

Despite the use of current advanced therapies in wound healing, there are still some wounds which fail to heal (21). In the present study a collagen chitosan scaffold was fabricated to treat skin wounds in a rat model and these scaffolds were loaded either with bone marrow derived stem cells or concentrated conditioned media to assess their effect on the healing time and quality of the healed skin.

In the present study a rat model has been used since it is the most usually in vivo model used although porcine skin shows more similarities to the human skin regarding the skin healing time and wound traction in accordance to Ferreira \& Gomes (22).

In accordance to Chandraker et al., (23) autografts have been the gold standard for treating skin defects. It has been shown that this treatment regimen has a lot of risks including long healing time, low survival rate, limited source and new defect of the donor site. In response to these defects a collagen chitosan scaffold has been fabricated in the present work.

Natural and synthetic materials have been used to fabricate biomedical scaffolds. Although synthetic materials have excellent mechanical properties and are easily processed but they have a major drawback which is the low biological activity in contrast to the natural materials as stated by Zahedi et al., (24). Therefore, in the current study natural materials (collagen and chitosan) have been chosen to fabricate the scaffold.

Different methods and sources have been recorded in the literatures $(25-27)$ in which extracted collagen was retrieved in a time interval ranging from days to weeks in contrast to the present study which extracted collagen in less than 3 hours using a simple procedure as stated by Pacak et al., (28).

Collagen has been chosen as the main ingredient in the fabricated scaffold in the current study due to its remarkable biocompatibility, water holding capacity, biodegradability and its ability to regulate the cellular growth, differentiation and proliferation. However, scaffolds containing collagen are known for their poor mechanical performance and its degradation within 2 to 3 days in the body temperature in accordance to $\mathrm{Li}$ et al., (29).

Therefore to improve the properties of collagen scaffolds they have been crosslinked with chitosan using glutaraldehyde cross linking agent to form a natural composite scaffold having mechanical properties similar to that of the soft tissues in accordance to Mighri et al., (30) and to blend perfectly and homogenously with it in terms of pore size and distribution as stated by Bianchera et al., (31). Chitosan has been chosen as a favorable candidate for tissue engineered scaffolds in the present study due to its bactericidal property as it inhibits the growth of many types of bacteria, fungi and yeast beside its biocompatibility, cytocompatibility, cost effectiveness, mechanical stability and good film forming capacity in accordance to Mighri et al., (30). 
This antibacterial property is of important significance in the present work as it removes the bacteria which might be the reason for delayed wound healing or pathogen resistance.

In a study conducted by Haifei et al, supporting the results of the current study, there was a decrease in the healing time concerning the collagen chitosan scaffold in comparison to the control group (32).

In the current study, Scaffold treated group SG had shown an accelerated wound healing in comparison to control untreated group, with enhanced macrophage recruitment 48 hours after injury and which are known to dominate during the pro-inflammatory phase of wound healing. In addition, the collagen deposition in the completely healed wound was also significantly higher in SG in comparison to control group which denotes the efficient formation of ECM needed for remodeling

In another study conducted by Romanova et al., (33) it has been proven that chitosan collagen scaffolds with micro fibrous architecture decreased the healing time for a skin defect in a rat model in comparison to the control group which agrees with the results of the current study. They concluded that thinner collagen chitosan scaffolds less than $0.5 \mathrm{~mm}$ enhanced collagen remodeling and promoted earlier angiogenesis as stated by the present study. In the same study thicker collagen chitosan scaffolds were fabricated and assessed. The drawbacks of thick scaffolds is that they disable the oxygen diffusion through the graft and into the tissues leading to complex problems which mainly include tissue necrosis, acanthosis, tissue degeneration, inflammation and immune reactions which contrasts with the present study. Therefore, the thickness of the collagen chitosan scaffold is of extreme importance regarding the wound healing process.

In a study conducted by Mehanna et al., (34) who used fibrin glue as a treatment regimen for a full thickness wound defect and revealed no significant difference in the duration taken for complete healing of the wounds between the control and the fibrin glue treated group ,which all extended for more than average of 30 days. That is to say that the factor which attributed to accelerated healing is the nature of the scaffold.

\section{CONCLUSION}

Collagen chitosan scaffold is a potential dermal substitute offering lower healing times in skin defects in a rat model

\section{CONFLICT OF INTEREST}

No potential conflict of interest relevant to this article was reported.

\section{REFERENCES}

1. Hölzle F, Mohr C, Wolff KD. Reconstructive oral and maxillofacial surgery. Deutsches Ärzteblatt International. 2008 Nov;105(47):815.

2. Feinberg SE, Aghaloo TL, Cunningham Jr LL. Role of Tissue Engineering in Oral and Maxillofacial
Reconstruction: Findings of the 2005 AAOMS Research Summit. J Oral Maxillofac Surg. 2005;63:1418- 25.

3. Wang X, Li Q, Hu X, Ma L, You C, Zheng Y, et al. Fabrication and characterization of poly(L-lactide-coglycolide) knitted mesh-reinforced collagen-chitosan hybrid scaffolds for dermal tissue engineering. J Mech Behav Biomed Mater. 2012;8:204-15.

4. Balasubramani M, Kumar TR, Babu M. Skin substitutes: a review. Burns. 2001;27:534-44.

5. Zhao S, Li L, Wang H, Zhang Y, Cheng X, Zhou N, Rahaman $M N$, et al. Wound dressings composed of copper-doped borate bioactive glass microfibers stimulate angiogenesis and heal full-thickness skin defects in a rodent model. Biomaterials. 2015;53:379-91.

6. Wang X, You C, Hu X, Zheng Y, Li Q, Feng Z, Sun H, et al. The roles of knitted mesh-reinforced collagenchitosan hybrid scaffold in the one-step repair of fullthickness skin defects in rats. Acta Biomater. 2013;9:7822-32.

7. Madhumathi K, Sudheesh Kumar PT, Abhilash S, Sreeja $\mathrm{V}, \quad$ Tamura $\mathrm{H}$, Manzoor $\frac{\mathrm{K}}{\mathrm{K}}$ et al. Development of novel chitin/nanosilver composite scaffolds for wound dressing applications. J Mater Sci Mater Med. 2010;21:807-13.

8. Wiegand C, Heinze T, Hipler UC. Comparative in vitro study on cytotoxicity, antimicrobial activity, and binding capacity for pathophysiological factors in chronic wounds of alginate and silver-containing alginate. Wound Repair Regen. 2009;17:511-21.

9. Burgeson RE, Nimni ME. Collagen types. Molecular structure and tissue distribution. Clin Orthop Relat Res. 1992;282:250-72.

10. Chevallay B, Herbage D. Collagen-based biomaterials as 3D scaffold for cell cultures: Applications for tissue engineering and gene therapy. Med Biol Eng Comput. 2000;38:211-8.

11. Wolf K, Alexander S, Schacht V, Coussens LM, von $\frac{\text { Andrian }}{\mathrm{UH}}, \frac{\mathrm{van}}{\text { Rheenen }}$ et al. Collagen-based cell migration models in vitro and in vivo. Semin Cell Dev Biol. 2009;20:931-41.

12. Croisier F, Jérôme C. Chitosan-based biomaterials for tissue engineering. Eur Polym J. 2013;49:780-92.

13. Dhandayuthapani B, Krishnan UM, Sethuraman S. Fabrication and characterization of chitosan-gelatin blend nanofibers for skin tissue engineering. J Biomed Mater Res B Appl Biomater. 2010;94:264-72.

14. Aranaz I, Mengíbar M, Harris R, Paños I, Miralles B. Functional characterization of chitin and chitosan. Curr Chem Biol. 2009;3:203-30.

15. Yang Y, Xia T, Zhi W, Wei L, Weng J. Promotion of skin regeneration in diabetic rats by electrospun coresheath fibers loaded with basic fibroblast growth factor. Biomaterials. 2011;32:4243-54.

16. Songjiang Z, Lixiang W. Amyloid-beta associated with chitosan nano-carrier has favorable immunogenicity and 
permeates the BBB. AAPS Pharm Sci Tech. 2009;10:900-5.

17. Pacak CA, 只wers JM, Cowan DB. Ultrarapid Purification of Collagen Type I for Tissue Engineering Applications. Tissue Eng Part C Methods. 2011;17:87985.

18. de Queiroz Antonino R, Lia Fook B, de Oliveira Lima V, de Farias Rached R, Lima E, da Silva Lima R, Peniche Covas C, Lia Fook M. Preparation and characterization of chitosan obtained from shells of shrimp (Litopenaeus vannamei Boone). Marine drugs. 2017 May;15(5):14152.

19. Han C, Zhang L, Sun J, Shi H, Zhou J, Gao C. Application of collagen-chitosan/fibrin glue asymmetric scaffolds in skin tissue engineering. J Zhejiang Univ Sci B. 2010;11:524-30.

20. Pereira T, Armada-da Silva PA, Amorim I, Rêma A, Caseiro AR, Gärtner A, et al. Effects of human mesenchymal stem cells isolated from Wharton's jelly of the umbilical cord and conditioned media on skeletal muscle regeneration using a myectomy model. Stem Cells Int. 2014;2014:1-16.

21. Zhao S, Li L, Wang H, Zhang Y, Cheng X, Zhou N, Rahaman MN, Liu Z, Huang W, Zhang C. Wound dressings composed of copper-doped borate bioactive glass microfibers stimulate angiogenesis and heal fullthickness skin defects in a rodent model. Biomaterials. 2015 Jun 1;53:379-91.

22. Ferreira AD, Gomes DA. Stem cell extracellular vesicles in skin repair. Bioengineering. 2019 Mar;6(1):4.

23. Chandraker A, Arscott R, Murphy G, Lian C, Bueno E, Marty F, et al. Face transplantation in a highly sensitized recipient. Mil Med. 2016;181:221-6.

24.Zahedi E, Esmaeili A, Eslahi N, Shokrgozar M, Simchi A. Fabrication and Characterization of Core-Shell Electrospun Fibrous Mats Containing Medicinal Herbs for Wound Healing and Skin Tissue Engineering. Marine drugs. 2019 Jan;17(1):27-40.

25. Blanco M, Vázquez JA, Pérez-Martín RI, G Sotelo C. Collagen Extraction Optimization from the Skin of the Small-Spotted Catshark (S. canicula) by Response Surface Methodology. Marine drugs. 2019 Jan;17(1):40.
26. Rajan N, Habermehl J, Coté MF, Doillon CJ, Mantovani D. Preparation of ready-to-use, storable and reconstituted type I collagen from rat tail tendon for tissue engineering applications. Nat Protoc. 2006;1:2753-8.

27. Yang $H$, Shu Z. The extraction of collagen protein from pigskin. J Chem Pharm Res. 2014;6:683-7.

28. Pacak CA, MacKay AA, Cowan DB. An improved method for the preparation of type I collagen from skin. J Vis Exp. 2014;83:e51011.

29. Li J, Zhou C, Luo C, Qian B, Liu S, Zeng Y, et al. Nacetyl cysteine-loaded graphene oxide-collagen hybrid membrane for scarless wound healing. Theranostics. 2019;9:5839-53.

30. Mighri N, Mao J, Mighri F, Ajji A, Rouabhia M. Chitosan-coated collagen membranes promote chondrocyte adhesion, growth, and interleukin-6 secretion. Materials. 2015;8(11):7673-89.

31. Bianchera A, Salomi E, Pezzanera M, Ruwet E, Bettini R, Elviri L. Chitosan hydrogels for chondroitin sulphate controlled release: an analytical characterization. Journal of analytical methods in chemistry. 2014;2014.

32. Haifei S, Xingang W, Shoucheng W, Zhengwei M, Chuangang $\mathrm{Y}$, Chunmao $\mathrm{H}$. The effect of collagenchitosan porous scaffold thickness on dermal regeneration in a one-stage grafting procedure. J Mech Behav Biomed Mater. 2014;29:114-25.

33. Romanova OA, Grigor'ev TE, Goncharov ME, Rudyak SG, Solov'yova EV, Krasheninnikov ST, Saprykin VP, Sytina EV, Chvalun SN, Pal'tsev MA, Panteleev AA. Chitosan as a modifying component of artificial scaffold for human skin tissue engineering. Bulletin of experimental biology and medicine. 2015 Aug 1;159(4):557-66.

34. Mehanna RA, Nabil I, Attia N, Bary AA, Razek KA, Ahmed TA, Elsayed F. The effect of bone marrowderived mesenchymal stem cells and their conditioned media topically delivered in fibrin glue on chronic wound healing in rats. BioMed research international. 2015;2015. 